\title{
A Generalization of the Krätzel Function and Its Applications
}

\author{
Neşe Dernek, ${ }^{1}$ Ahmet Dernek, ${ }^{1}$ and Osman Yürekli ${ }^{2}$ \\ ${ }^{1}$ Department of Mathematics, University of Marmara, Istanbul, Turkey \\ ${ }^{2}$ Department of Mathematics, Ithaca College, Ithaca, NY 14850, USA
}

Correspondence should be addressed to Osman Yürekli; yurekli@ithaca.edu

Received 26 July 2016; Revised 26 December 2016; Accepted 5 January 2017; Published 26 January 2017

Academic Editor: Ming-Sheng Liu

Copyright (c) 2017 Neşe Dernek et al. This is an open access article distributed under the Creative Commons Attribution License, which permits unrestricted use, distribution, and reproduction in any medium, provided the original work is properly cited.

In this paper, we introduce new functions $Y_{\rho, r}^{v}(x)$ as a generalization of the Krätzel function. We investigate recurrence relations, Mellin transform, fractional derivatives, and integral of the function $Y_{\rho, r}^{v}(x)$. We show that the function $Y_{\rho, r}^{v}(x)$ is the solution of differential equations of fractional order.

\section{Introduction}

The Krätzel function is defined for $x>0$ by the integral

$$
Z_{\rho}^{\nu}(x)=\int_{0}^{\infty} t^{\nu-1} e^{-t^{\rho}-x / t} d t
$$

where $\rho \in \mathbb{R}$ and $\nu \in \mathbb{C}$, such that $\mathfrak{R}(\nu)<0$ for $\rho \leq 0$ (cf. [1]). For $\rho \geq 1$ the function (1) was introduced by Krätzel as a kernel of the integral transform as follows:

$$
\left(\mathscr{K}_{\nu}^{\rho} f\right)(x)=\int_{0}^{\infty} Z_{\rho}^{v}(x t) f(t) d t \quad(x>0) .
$$

The Krätzel function $Z_{\rho}^{v}(x)$ is related to the modified Bessel function of the second kind $K_{v}$ by the relationship

$$
Z_{1}^{v}(x)=2 x^{v / 2} K_{v}(2 \sqrt{x})
$$

The generalized Krätzel function $D_{\rho, r}^{\nu, \alpha}(x)$ is given in [2,3] by the following relation:

$$
D_{\rho, r}^{\nu, \alpha}(x)=\int_{0}^{\infty} t^{\nu-1}\left[1+a(\alpha-1) t^{\rho}\right]^{1 /(\alpha-1)} e^{-x t^{-r}} d t
$$

where $\rho \in \mathbb{R}, r \in \mathbb{R}^{+}, v \in \mathbb{C}$, and $\alpha>1$. Kilbas and Kumar considered the special case for $r=1$ in [2], calculated fractional derivatives and fractional integrals of $D_{\rho, 1}^{\nu, \alpha}(x)$, and obtained a representation using Wright hypergeometric functions. On the other hand the general case of (1) is given in [2, (54), p. 845].

We consider the generalized Krätzel function $Y_{\rho, r}^{v}(x)$ defined by the integral

$$
Y_{\rho, r}^{\nu}(x)=\int_{0}^{\infty} t^{\nu-1} e^{-t^{\rho}-x t^{-r}} d t
$$

for $x>0, \rho \in \mathbb{R}, r \in \mathbb{R}^{+}$, and $\nu \in \mathbb{C}$. The function $Y_{\rho, r}^{\nu}(x)$ is a generalization of the Krätzel function $Z_{\rho}^{\nu}(x)$ since

$$
\lim _{r \rightarrow 1} Y_{\rho, r}^{v}(x)=Z_{\rho}^{v}(x) .
$$

If $a=1$ in (4), then

$$
\lim _{\alpha \rightarrow 1} D_{\rho, r}^{\nu, \alpha}(x)=Y_{\rho, r}^{\nu}(x) .
$$

We give some definitions and inequalities that will be needed. The Turán type inequalities

$$
f_{n}(x) \cdot f_{n+2}(x)-\left[f_{n-1}(x)\right]^{2} \geq 0, \quad n=0,1,2, \ldots
$$

are important and well known in many fields of mathematics (cf. [4]). A function $f(x)$ is completely monotonic on $(0, \infty)$, if $f$ has derivatives of all orders and satisfies the inequality

$$
(-1)^{m} f^{(m)}(x) \geq 0
$$


for all $x>0$ and $m \in \mathbb{N}$ (cf. [5, Section IV]). A function $f(x)$ is said to be log-convex on $(0, \infty)$, if

$$
f\left[\alpha x_{1}+(1-\alpha) x_{2}\right] \leq\left[f\left(x_{1}\right)\right]^{\alpha}\left[f\left(x_{2}\right)\right]^{1-\alpha}
$$

for all $x_{1}, x_{2}>0$ and $\alpha \in[0,1]$ (cf. [5, p. 167]).

Let $p, q \in \mathbb{R}$ such that $p>1$ and $1 / p+1 / q=1$. If $f$ and $g$ are real valued functions defined on a closed interval and $|f|^{p},|g|^{q}$ are integrable in this interval, then we have

$$
\begin{aligned}
& \int_{a}^{b}|f(t) g(t)| d t \\
& \quad \leq\left[\int_{a}^{b}|f(t)|^{p} d t\right]^{1 / p}\left[\int_{a}^{b}|g(t)|^{q} d t\right]^{1 / q} .
\end{aligned}
$$

The following inequality is due to Mitrinović et al. (cf. [6, p. 239]). Let $f$ and $g$ be two functions which are integrable and monotonic in the same sense on $[a, b]$ and $p$ is a positive and integrable function on the same interval, then the following inequality holds true:

$$
\begin{aligned}
& \int_{a}^{b} p(t) f(t) d t \int_{a}^{b} p(t) g(t) d t \\
& \quad \leq \int_{a}^{b} p(t) d t \int_{a}^{b} p(t) f(t) g(t) d t,
\end{aligned}
$$

if and only if one of the functions $f$ and $g$ reduces to a constant.

The Mellin transform of the function $f$ is defined by

$$
\mathscr{M}\{f(x) ; s\}=\int_{0}^{\infty} x^{s-1} f(x) d x
$$

when $\mathscr{M}\{f(x) ; s\}$ exists. The Mellin transform of the generalized Krätzel function (5) is given by Kilbas and Kumar in [2].

The Laplace transform of the function $f$ is defined by

$$
\mathscr{L}\{f(x) ; s\}=\int_{0}^{\infty} e^{-s x} f(x) d x
$$

provided that the integral on the right-hand side exists.

The Liouville fractional integral is defined by

$$
\left(\mathscr{I}_{-}^{\alpha} f\right)(x)=\frac{1}{\Gamma(\alpha)} \int_{x}^{\infty}(t-x)^{\alpha-1} f(t) d t
$$

and its derivatives $\mathscr{I}_{-}^{\alpha}$ and $\mathscr{D}_{-}^{\alpha}$ are

$$
\begin{aligned}
& \left(\mathscr{D}_{-}^{\alpha} f\right)(x)=\left(-\frac{d}{d x}\right)^{[\Re(\alpha)]+1}\left(\mathscr{I}_{-}^{1-\alpha+[\Re(\alpha)]} f\right)(x) \\
& =\frac{1}{\Gamma(1-\alpha+[\Re(\alpha)])} \int_{x}^{\infty}(t-x)^{-\alpha+[\Re(\alpha)]} f(t) d t
\end{aligned}
$$

where $x>0, \alpha \in \mathbb{C}$, and $\mathfrak{R}(\alpha)>0$ (cf. [7, Section 5.1]).

We introduce new operators

$$
\begin{aligned}
\mathscr{L}_{\lambda}^{\nu}:= & -r x \mathscr{D}_{-}^{\lambda+1}+(\lambda r-\nu) \mathscr{D}_{-}^{\lambda} \\
\mathscr{T}_{\lambda}^{v}:= & r^{2} x^{2} \mathscr{D}_{-}^{2 \lambda+2}+r x(2 \nu-3 \lambda r-r) \mathscr{D}_{-}^{2 \lambda+1} \\
& +(\nu-r \lambda)(\nu-2 r \lambda) \mathscr{D}_{-}^{2 \lambda},
\end{aligned}
$$

where $\nu \in \mathbb{C}$ and $\lambda>0$.
A standard source in the theory of fractional calculus is the book [8]. For applications of fractional calculus to science and engineering, we refer the reader to the articles [9-11].

In this paper, we investigate the properties of the functions $Y_{\rho, r}^{v}(x)$ and prove their composition of $Y_{\rho, r}^{v}(x)$ with fractional integral and derivatives $\left(\mathscr{I}_{-}^{\alpha} f\right)(x),\left(\mathscr{D}_{-}^{\alpha} f\right)(x)$ given by (15) and (16) (cf. $[2,6,12,13])$. In Section 3, we show that $Y_{\rho, r}^{v}(x)$ is the solution of differential equations of fractional order.

\section{The Main Theorems}

In this section, we will give some properties of generalized Krätzel functions $Y_{\rho, r}^{v}$.

Lemma 1. Let $\rho \in \mathbb{R}(\rho \neq 0), r \in \mathbb{R}^{+}, \nu \in \mathbb{C}, \mathfrak{R}(s)>0$ be such that $\mathfrak{R}(\nu+r s)>0$ when $\rho>0$ and $\mathfrak{R}(\nu+r s)<0$ when $\rho<0$. The Mellin transform of the function $Y_{\rho, r}^{v}(x)$ is given by

$$
\mathscr{M}\left\{Y_{\rho, r}^{v} ; s\right\}=\frac{1}{|\rho|} \Gamma(s) \Gamma\left(\frac{\nu+r s}{\rho}\right) \quad x>0 .
$$

Proof. Using (13) and (5), we have

$$
\mathscr{M}\left\{Y_{\rho, r}^{\nu} ; s\right\}=\int_{0}^{\infty} x^{s-1}\left(\int_{0}^{\infty} t^{\nu-1} e^{-t^{\rho}-x t^{-r}} d t\right) d x
$$

Changing the order of integration and using the substitution of $x t^{-r}=u$, we have

$$
\begin{aligned}
\mathscr{M}\left\{Y_{\rho, r}^{\nu} ; s\right\} & =\int_{0}^{\infty} t^{\nu-1} e^{-t^{\rho}}\left(\int_{0}^{\infty} x^{s-1} e^{-x t^{-r}} d x\right) d t \\
& =\Gamma(s) \int_{0}^{\infty} t^{\nu+r s-1} e^{-t^{\rho}} d t .
\end{aligned}
$$

Making the change of variable the integral $t^{\rho}=z$, and using the known formula (1) from [14, p. 145], we find that

$$
\begin{aligned}
\mathscr{M}\left\{Y_{\rho, r}^{v} ; s\right\} & =\frac{\Gamma(s)}{\rho} \int_{0}^{\infty} z^{(\nu+r s) / \rho-1} e^{-z} d z \\
& =\frac{\Gamma(s)}{\rho} \Gamma\left(\frac{\nu+r s}{\rho}\right),
\end{aligned}
$$

when $\rho>0$ and

$$
\begin{aligned}
\mathscr{M}\left\{Y_{\rho, r}^{\nu} ; s\right\} & =\frac{\Gamma(s)}{\rho} \int_{0}^{\infty} z^{(\nu+r s) / \rho-1} e^{-z} d z \\
& =-\frac{\Gamma(s)}{\rho} \Gamma\left(\frac{\nu+r s}{\rho}\right),
\end{aligned}
$$

when $\rho<0$.

Theorem 2. We have the following relationship for the function $Y_{\rho, r}^{v}(x)$ :

$$
Y_{\rho, r}^{v}(x)=L\left\{\frac{1}{r} t^{-v / r-1} e^{-t^{-\rho / r}} ; x\right\}
$$

where $\rho \in \mathbb{R}, r \in \mathbb{R}^{+}, v \in \mathbb{C}$, and $x>0$. 
Proof. Using (5) and making the change of $t^{-r}=u$, we obtain

$$
\begin{aligned}
Y_{\rho, r}^{\nu}(x) & =\int_{0}^{\infty} t^{\nu-1} e^{-t^{\rho}-x t^{-r}} d t \\
& =\int_{0}^{\infty} \frac{1}{r} u^{-\nu / r-1} e^{-u^{-\rho / r}} e^{-x u} d u .
\end{aligned}
$$

Now the assertion (24) follows from the definition (14) of the Laplace transform.

Using the known formula (29) from [14, p. 146], we find that

$$
\begin{aligned}
Y_{\rho, \rho}^{\nu}(x) & =\frac{1}{\rho} \mathscr{L}\left\{t^{-v / r-1} e^{-t^{-\rho / r}} ; x\right\} \\
& =\frac{2}{\rho} x^{\nu / 2 \rho} K_{\nu / \rho}(2 \sqrt{x}),
\end{aligned}
$$

for $\rho=1$ :

$$
\begin{aligned}
Y_{1,1}^{\nu}(x) & =Z_{1}^{v}(x)=2 x^{\nu / 2} K_{\nu}(2 \sqrt{x}), \\
Y_{1 / r, 1 / r}^{v}(x) & =2 r x^{\nu / 2} K_{\nu}(2 \sqrt{x}) .
\end{aligned}
$$

Theorem 3. If $\rho \in \mathbb{R}, r \in \mathbb{R}^{+}, \nu \in \mathbb{C}$ and $x>0$, then the following assertions are true:

(a) The function $Y_{\rho, r}^{v}(x)$ satisfies the recurrence relation

$$
\nu Y_{\rho, r}^{\nu}(x)=\rho Y_{\rho, r}^{\nu+\rho}(x)-r x Y_{\rho, r}^{\nu-r}(x)
$$

(b) The function $x \rightarrow Y_{\rho, r}^{v}(x)$ is completely monotonic on $(0, \infty)$.

Proof. (a) The above recurrence relation could be verified by using integration by parts as follows:

$$
\begin{aligned}
Y_{\rho, r}^{\nu}(x)= & \left.\frac{1}{v} t^{\nu} e^{-t^{\rho}-x t^{-r}}\right|_{0} ^{\infty} \\
& -\int_{0}^{\infty} \frac{1}{\nu} t^{\nu}\left[-\rho t^{\rho-1}+r x t^{-r-1}\right] e^{-t^{\rho}-x t^{-r}} d t \\
= & \frac{\rho}{v} \int_{0}^{\infty} t^{\nu+\rho-1} e^{-t^{\rho}-x t^{-r}} d t \\
& -\frac{r x}{\nu} \int_{0}^{\infty} t^{\nu-r-1} e^{-t^{\rho}-x t^{-r}} d t \\
= & \frac{\rho}{v} Y_{\rho, r}^{\nu+\rho}(x)-\frac{r x}{\nu} Y_{\rho, r}^{\nu-r}(x) .
\end{aligned}
$$

(b) From Bernstein-Widder theorem (see Theorem 1, [5, p. 145]), the function $Y_{\rho, r}^{v}(x)$ is completely monotonic on $(0, \infty)$ for all $x>0$. This could be verified directly as follows:

$$
\frac{d^{n}}{d x^{n}} Y_{\rho, r}^{\nu}(x)=(-1)^{n-1} Y_{\rho, r}^{\nu-n r}(x)>0
$$

which follows via mathematical induction from (5) provided that $\rho \in \mathbb{R}, r \in \mathbb{R}^{+}, \nu \in \mathbb{C}$ and $x>0$. From BernsteinWidder theorem, generalized forms of Krätzel function are completely monotonic on $(0, \infty)$ for all $x>0$. Due to $(30)$, the functions are completely monotonic on $(0, \infty)$ for all $x>$ 0 .

Setting $r \rightarrow 1$ and using (28), the equation yields

$$
Y_{\rho, 1}^{\nu}(x)=\frac{\rho}{\nu} Y_{\rho, 1}^{\nu+\rho}(x)-\frac{x}{\nu} Y_{\rho, 1}^{\nu-1}(x) .
$$

Then using (31) and (6), we obtain the relation

$$
v Z_{\rho}^{\nu}(x)=\rho Z_{\rho}^{\nu+\rho}(x)-x Z_{\rho}^{\nu-1}(x),
$$

(cf. 2.1 of Theorem 1 from [12]).

Theorem 4. Let $v_{1}, v_{2}, \rho \in \mathbb{R}, 0<\lambda<1$, and $x>0$, then the following assertions hold true:

(a) The function $v \rightarrow Y_{\rho, r}^{v}(x)$ is log-convex on $\mathbb{R}:$

$$
Y_{\rho, r}^{\lambda \nu_{1}+(1-\lambda) v_{2}, \alpha}(x) \leq\left[Y_{\rho, r}^{\nu_{1}}(x)\right]^{\lambda}\left[Y_{\rho, r}^{\nu_{2}}(x)\right]^{1-\lambda}
$$

(b) The function $x \rightarrow Y_{\rho, r}^{v}(x)$ is log-convex on $(0, \infty)$ :

$$
Y_{\rho, r}^{\nu}\left(\lambda x_{1}+(1-\lambda) x_{2}\right) \leq\left[Y_{\rho, r}^{v}\left(x_{1}\right)\right]^{\lambda}\left[Y_{\rho, r}^{\nu}\left(x_{2}\right)\right]^{1-\lambda} \text {. }
$$

(c) The function $Y_{\rho, r}^{v}(x)$ satisfies the following relation:

$$
Y_{\rho, r}^{\nu}\left(t^{r}\right)=t^{\nu} Y_{r, \rho}^{-\nu}\left(t^{\rho}\right)
$$

Proof. (a) Using (5) and (11), we obtain

$$
\begin{aligned}
& Y_{\rho, r}^{\lambda \nu_{1}+(1-\lambda) \nu_{2}}(x)=\int_{0}^{\infty} t^{\lambda \nu_{1}+(1-\lambda) \nu_{2}-1} e^{-t^{\rho}-x t^{-r}} d t \\
& \quad=\int_{0}^{\infty}\left(t^{\nu_{1}-1} e^{-t^{\rho}} e^{-x t^{-r}}\right)^{\lambda}\left(t^{\nu_{2}-1} e^{-t^{\rho}} e^{-x t^{-r}}\right)^{1-\lambda} d t \\
& \quad \leq\left[\int_{0}^{\infty} t^{\nu_{1}-1} e^{-t^{\rho}-x t^{-r}} d t\right]^{\lambda}\left[\int_{0}^{\infty} t^{\nu_{2}-1} e^{-t^{\rho}-x t^{-r}} d t\right]^{1-\lambda} \\
& \quad=\left[Y_{\rho, r}^{\nu_{1}}(x)\right]^{\lambda}\left[Y_{\rho, r}^{\nu_{2}}(x)\right]^{1-\lambda},
\end{aligned}
$$

where $\lambda \in[0,1], \nu_{1}, \nu_{2}, \rho \in \mathbb{R}, \alpha>1$, and $x>0$. Thus, $\nu \rightarrow Y_{\rho, r}^{\nu}(x)$ is log-convex on $\mathbb{R}$.

(b) The integrand in (5) is a log-linear convex function of $x$. By using (11), we have

$$
\begin{aligned}
& Y_{\rho, r}^{\nu}\left(\lambda x_{1}+(1-\lambda) x_{2}\right) \\
& =\int_{0}^{\infty} t^{\nu-1} e^{-t^{\rho}} e^{-\left(\lambda x_{1}+(1-\lambda) x_{2}\right) t^{-r}} d t \\
& =\int_{0}^{\infty}\left[t^{\nu-1} e^{-t^{\rho}} e^{-x_{1} t^{-r}}\right]^{\lambda}\left[t^{\nu-1} e^{-t^{\rho}} e^{-x_{2} t^{-r}}\right]^{1-\lambda} d t \\
& \leq\left[\int_{0}^{\infty} t^{\nu-1} e^{-t^{\rho}} e^{-x_{1} t^{-r}} d t\right]^{\lambda}\left[\int_{0}^{\infty} t^{\nu-1} e^{-t^{\rho}} e^{-x_{2} t^{-r}} d t\right]^{1-\lambda} \\
& =\left[Y_{\rho, r}^{\nu}\left(x_{1}\right)\right]^{\lambda}\left[Y_{\rho, r}^{\nu}\left(x_{2}\right)\right]^{1-\lambda},
\end{aligned}
$$

$$
(n=0,1,2, \ldots)
$$


where $\lambda \in[0,1], \nu, \rho \in \mathbb{R}, r>0$, and $x_{1}, x_{2}>0$. Thus, $x \rightarrow Y_{\rho, r}^{v}(x)$ is log-convex on $(0, \infty)$.

(c) Again using (5), we conclude that

$$
\begin{aligned}
& Y_{\rho, r}^{\nu}(x)=\int_{0}^{\infty} t^{\gamma-1} e^{-t^{\rho}-x t^{-r}} d t, \quad\left(t=x^{1 / r} u^{-1}\right) \\
& \quad=\int_{0}^{\infty}\left(x^{1 / r} u^{-1}\right)^{\nu-1} e^{-\left(x^{1 / r} u^{-1}\right)^{\rho}} e^{-x\left(x^{1 / r} u^{-1}\right)^{-r}} x^{1 / r} u^{-2} d u \\
& =x^{\nu / r} \int_{0}^{\infty} u^{-\nu-1} e^{-u^{r}-x^{\rho / r} u^{-\rho}} d u \\
& =x^{\nu / r} Y_{r, \rho}^{-\nu}\left(x^{\rho / r}\right)
\end{aligned}
$$

or for the change of $x=t^{r}$, we obtain (35).

Moreover, since $Y_{\rho, r}^{v}(x)$ is log-convex on $\mathbb{R}$, we have Turán type inequality

$$
\left[Y_{\rho, r}^{\left(v_{1}+v_{2}\right) / 2}(x)\right]^{2} \leq Y_{\rho, r}^{v_{1}}(x) Y_{\rho, r}^{\nu_{2}}(x)
$$

for $v_{1}, v_{2}, \rho \in \mathbb{R}, \alpha>1$, and $x>0$. Making the change of variable $v_{1}=v-2$ and $v_{2}=v$, the equation yields

$$
f_{\rho}^{\nu, \alpha}(x)=\left[Y_{\rho, r}^{\nu-1}(x)\right]^{2}-Y_{\rho, r}^{\nu-2}(x) Y_{\rho, r}^{\nu}(x) \leq 0
$$

which is valid for $v, \rho \in \mathbb{R}, \alpha>1$, and $x>0$.

Using (39) and making the change of variables $\nu_{1}=v-$ $n-1$ and $\nu_{2}=v-n+1$, we have

$$
\left[Y_{\rho, r}^{\nu-n}(x)\right]^{2} \leq Y_{\rho, r}^{\nu-n-1}(x) Y_{\rho, r}^{\nu-n+1}(x) .
$$

Theorem 5. If $\nu, \rho \in \mathbb{R}, r \in \mathbb{R}^{+}$and $x>0$, then the following inequality holds true:

$$
\Gamma\left(\frac{\nu}{r}\right) Y_{\rho / r, 1}^{-1 / r}(x) \leq x^{(\nu-1) / r} \Gamma\left(\frac{1}{r}\right) Y_{\rho, r}^{-\nu}(x) .
$$

Proof. Let $p(t)=e^{-x t^{r}}, f(t)=t^{\nu-1}$ and $g(t)=e^{-t^{-\rho}}$. The function $f(t)$ is increasing on $(0, \infty)$ for $v \geq 1$ and is decreasing for $\nu \leq 1$. On the other hand, we observe that, for all $\rho>0$,

$$
\frac{g^{\prime}(t)}{g(t)}=\rho t^{-\rho-1}>0
$$

Thus, $g(t)$ is increasing if and only if $\rho>0$. Moreover, making the change of $t^{r}=u$ and using the known formula (1) from [14, p. 137], we have

$$
\int_{0}^{\infty} p(t) d t=\int_{0}^{\infty} e^{-x t^{r}} d t=\frac{1}{r} \Gamma\left(\frac{1}{r}\right) x^{-1 / r} .
$$

Making the change of $t^{-r}=u$, we find

$$
\int_{0}^{\infty} p(t) f(t) d t=\int_{0}^{\infty} e^{-x t^{r}} t^{\nu-1} d t=\frac{1}{r} \Gamma\left(\frac{\nu}{r}\right) x^{-v / r} .
$$

Making the change of variable $t=u^{-1 / r}$ and using (6), we have

$$
\begin{aligned}
\int_{0}^{\infty} p(t) g(t) d t & =\int_{0}^{\infty} e^{-x t^{r}} e^{-t^{-\rho}} d t \\
& =\frac{1}{r} \int_{0}^{\infty} u^{-1 / r-1} e^{-u^{\rho / r}} e^{-x / u} d u \\
& =\frac{1}{r} Y_{\rho / r, 1}^{-1 / r}(x) .
\end{aligned}
$$

Using (5) and making the change of variable $t=u^{-1}$, we find

$$
\begin{gathered}
\int_{0}^{\infty} p(t) f(t) g(t) d t=\int_{0}^{\infty} e^{-x t^{r}} t^{\nu-1} e^{-t^{-\rho}} d t \\
=\int_{0}^{\infty} u^{-\nu-1} e^{-u^{\rho}-x u^{-r}} d u=Y_{\rho, r}^{-\nu}(x) .
\end{gathered}
$$

Finally, by using the relation (12), we obtain the inequality (42):

$$
\begin{aligned}
\int_{0}^{\infty} & p(t) f(t) d t \int_{0}^{\infty} p(t) g(t) d t \\
\leq & \int_{0}^{\infty} p(t) d t \int_{0}^{\infty} p(t) f(t) g(t) d t \frac{1}{r} \Gamma\left(\frac{\nu}{r}\right) x^{-\nu / r} \\
& \cdot \frac{1}{r} Y_{\rho / r, 1}^{-1 / r}(x) \\
\leq & \frac{1}{r} \Gamma\left(\frac{1}{r}\right) x^{-1 / r} \cdot Y_{\rho, r}^{-\nu}(x) x^{(\nu-1) / r} \Gamma\left(\frac{1}{r}\right) Y_{\rho, r}^{-\nu}(x) \\
\geq & \Gamma\left(\frac{\nu}{r}\right) Y_{\rho / r, 1}^{-1 / r}(x) .
\end{aligned}
$$

If we choose $r \rightarrow 1$ in (42), then we have

$$
x^{\nu-1} Y_{\rho, 1}^{-v}(x) \geq \Gamma(\nu) Y_{\rho, 1}^{-1}(x) .
$$

As a result, we find the following inequality by using (6):

$$
Z_{\rho}^{-v}(x) \geq x^{1-\nu} \Gamma(\nu) Z_{\rho}^{-1}(x) .
$$

\section{Differential Equations of Fractional Order}

In this section, we show that $Y_{\rho, r}^{v}(x)$ is the solution of differential equations of fractional order.

Theorem 6. If $\alpha, \nu \in \mathbb{C}, \mathfrak{R}(\alpha)>0$, and $\rho>0$, then the following identity holds true:

$$
\left(\mathscr{I}_{-}^{\alpha} Y_{\rho, r}^{\nu}\right)(x)=Y_{\rho, r}^{v+r \alpha}(x) .
$$

Proof. Applying (15), (5), and relation (11) of [15, p. 202], we obtain

$$
\begin{aligned}
& \left(\mathcal{F}_{-}^{\alpha} Y_{\rho, r}^{\nu}\right)(x) \\
& =\frac{1}{\Gamma(\alpha)} \int_{x}^{\infty}(t-x)^{\alpha-1} d t \int_{0}^{\infty} u^{\nu-1} e^{-u^{\rho}-t u^{-r}} d u \\
& =\int_{0}^{\infty} u^{\nu-1} e^{-u^{\rho}}\left(\frac{1}{\Gamma(\alpha)} \int_{x}^{\infty}(t-x)^{\alpha-1} e^{-t u^{-r}} d t\right) d u
\end{aligned}
$$




$$
\begin{aligned}
& =\int_{0}^{\infty} u^{\nu-1} e^{-u^{\rho}}\left(\mathscr{I}_{-}^{\alpha} e^{-t u^{-r}}\right)(x) d u \\
& =\int_{0}^{\infty} u^{\nu-1} e^{-u^{\rho}} e^{-x u^{-r}} u^{r \alpha} d u \\
& =\int_{0}^{\infty} u^{\nu+r \alpha-1} e^{-u^{\rho}} e^{-x u^{-r}} d u=Y_{\rho, r}^{\nu+r \alpha}(x) .
\end{aligned}
$$

Theorem 7. If $\alpha, v \in \mathbb{C}, \mathfrak{R}(\alpha)>0$, and $\rho>0$ then we have

$$
\left(\mathscr{D}_{-}^{\alpha} Y_{\rho, r}^{\nu}\right)(x)=Y_{\rho, r}^{\nu-r \alpha}(x) \text {. }
$$

Proof. Using (16), (5), and (51), we obtain

$$
\begin{aligned}
& \left(\mathscr{D}_{-}^{\alpha} Y_{\rho, r}^{\nu}\right)(x)=\left(-\frac{d}{d x}\right)^{[\Re(\alpha)]+1}\left(\mathscr{I}_{-}^{1-\alpha+[\Re(\alpha)]} Y_{\rho, r}^{\nu}\right)(x) \\
& =\left(-\frac{d}{d x}\right)^{[\Re(\alpha)]+1} Y_{\rho, r}^{\nu+r(1-\alpha+[\Re(\alpha)])}(x) \\
& =\left(-\frac{d}{d x}\right)^{[\Re(\alpha)]+1} \int_{0}^{\infty} t^{\nu+r(1-\alpha+[\Re(\alpha)])-1} e^{-t^{\rho}-x t^{-r}} d t \\
& =\int_{0}^{\infty} t^{\nu+r(1-\alpha+[\Re(\alpha)])-1} e^{-t^{\rho}}\left(-\frac{d}{d x}\right)^{[\Re(\alpha)]+1} \\
& \cdot\left(e^{-x t^{-r}}\right) d t=\int_{0}^{\infty} t^{\nu+r(1-\alpha+[\Re(\alpha)])-1} e^{-t^{\rho}} \\
& \cdot \frac{1}{t^{r([\Re(\alpha)]+1)}} e^{-x t^{-r}} d t=\int_{0}^{\infty} t^{\nu-\alpha r-1} e^{-t^{\rho}} e^{-x t^{-r}} d t \\
& =Y_{\rho, r}^{\nu-r \alpha}(x) .
\end{aligned}
$$

Corollary 8. If $\alpha, \beta$, and $v \in \mathbb{C}, \mathfrak{R}(\alpha)>0, \mathfrak{R}(\beta)>0$, and $\rho>0$, then we have

$$
\begin{aligned}
\left(\mathscr{D}_{-}^{\alpha} \mathscr{J}_{-}^{\beta} Y_{\rho, r}^{v}\right)(x) & =\left(\mathscr{I}_{-}^{\beta} \mathscr{D}_{-}^{\alpha} Y_{\rho, r}^{\nu}\right)(x) \\
& =Y_{\rho, r}^{\nu+\beta-\alpha+(1-r)(1+[\operatorname{Re}(\alpha)])} .
\end{aligned}
$$

Theorem 9. If $\nu \in \mathbb{C}$ and $\rho>0$, then the following identity holds true:

$$
\mathscr{L}_{\rho}^{\nu} Y_{\rho, r}^{\nu}(x)=-\rho Y_{\rho, r}^{\nu+(1-r) \rho}(x) .
$$

Proof. Applying (17) to (5), we get

$$
\begin{aligned}
\mathscr{L}_{\rho}^{\nu} Y_{\rho, r}^{\nu}(x) & =-r x \mathscr{D}_{-}^{\rho+1} Y_{\rho, r}^{\nu}(x)+(\rho r-\nu) \mathscr{D}_{-}^{\rho} Y_{\rho, r}^{\nu}(x) \\
& =-r x Y_{\rho, r}^{\nu-(\rho+1) r}(x)+(\rho r-\nu) Y_{\rho, r}^{\nu-\rho r}(x) \\
& =-r x \int_{0}^{\infty} t^{\nu-(\rho+1) r-1} e^{-t^{\rho}-x \mathrm{t}^{-r}} d t
\end{aligned}
$$

$$
\begin{aligned}
& +(\rho r-v) \int_{0}^{\infty} t^{\nu-\rho r-1} e^{-t^{\rho}-x t^{-r}} d t \\
= & -\int_{0}^{\infty} t^{\nu-\rho r-1}\left(r \frac{x}{t^{r}}+\nu-\rho r\right) e^{-t^{\rho}-x t^{-r}} d t .
\end{aligned}
$$

Using the formula

$$
\left(t^{\nu-\rho r} e^{-x t^{-r}}\right)^{\prime}=t^{\nu-\rho r-1}\left(\nu-\rho r+x r t^{-r}\right) e^{-x t^{-r}}
$$

and applying the integration by parts, we find

$$
\begin{aligned}
\mathscr{L}_{\rho}^{\nu} Y_{\rho, r}^{\nu}(x)= & -\int_{0}^{\infty}\left(t^{\nu-\rho r} e^{-x t^{-r}}\right)^{\prime} e^{-t^{\rho}} d t \\
= & -\left.t^{\nu-\rho r} e^{-x t^{-r}} e^{-t^{\rho}}\right|_{0} ^{\infty} \\
& +\int_{0}^{\infty} t^{\nu-\rho r} e^{-x t^{-r}}\left(-\rho t^{\rho-1} e^{-t^{\rho}}\right) d t \\
= & 0-\rho \int_{0}^{\infty} t^{\nu-\rho r+\rho-1} e^{-x t^{-r}} e^{-t^{\rho}} d t \\
= & -\rho Y_{\rho, r}^{\nu+(1-r) \rho}(x) .
\end{aligned}
$$

Corollary 10. If $v \in \mathbb{C}$ and $\rho>0$, then the function $Y_{\rho, r}^{v}(x)$ is a solution of the differential equation of fractional order

$$
\begin{gathered}
r x \mathscr{D}_{-}^{\rho+1} Y_{\rho, r}^{\nu}(x)+(\nu-\rho r) \mathscr{D}_{-}^{\rho} Y_{\rho, r}^{\nu}(x) \\
-\rho Y_{\rho, r}^{\nu+(1-r) \rho}(x)=0 .
\end{gathered}
$$

Remark 11. If $v \in \mathbb{C}$, and $\rho=r=1$, then the function $Y_{1,1}^{v}(x)=Z_{1}^{v}(x)$ is a solution of the following differential equation:

$$
x y^{\prime \prime}+(\nu-1) y^{\prime}-y=0
$$

(cf. $[13,(30)$, p. 20]).

Theorem 12. If $\nu \in \mathbb{C}$ and $\rho>0$, then the function $Y_{\rho, r}^{v}(x)$ is a solution of the differential equation of fractional order

$$
\begin{aligned}
& \left(\mathscr{T}_{\rho}^{\nu} Y_{\rho, r}^{\nu}\right)(x)+\rho^{2} Y_{\rho, r}^{\nu-2(r-1) \rho}(x) \\
& \quad-\rho^{2}(r-1) Y_{\rho, r}^{\nu-(2 r-1) \rho}(x)=0 .
\end{aligned}
$$

Proof. Using (18), (5), and (53), we get

$$
\begin{aligned}
& \left(\mathscr{T}_{\rho}^{\nu} Y_{\rho, r}^{\nu}\right)(x)=\int_{0}^{\infty}\left\{\frac{r^{2} x^{2}}{t^{2 r}}+(2 \nu-3 \rho r-r) \frac{r x}{t^{r}}\right\} \\
& \cdot t^{\nu-2 r \rho-1} e^{-x t^{-r}} e^{-t^{\rho}} d t+\int_{0}^{\infty}(\nu-r \rho)(\nu-2 r \rho) \\
& \cdot t^{\nu-2 r \rho-1} e^{-x t^{-r}} e^{-t^{\rho}} d t .
\end{aligned}
$$


If we take the derivative as the proof of Theorem 9, then we arrive at

$$
\begin{aligned}
& \left(\left(\nu-r \rho+\frac{r x}{t^{r}}\right) t^{\nu-2 r \rho} e^{-x t^{-r}}\right)^{\prime}=\left\{\frac{r^{2} x^{2}}{t^{2 r}}\right. \\
& \left.+(2 \nu-3 \rho r-r) \frac{r x}{t^{r}}+(\nu-r \rho)(\nu-2 r \rho)\right\} \\
& \cdot t^{\nu-2 r \rho-1} e^{-x t^{-r}} .
\end{aligned}
$$

Substituting (64), (16), into (63) and applying the integration by parts, we get

$$
\begin{aligned}
\left(\mathscr{T}_{\rho}^{\nu} Y_{\rho, r}^{\nu}\right)(x) \\
=\int_{0}^{\infty}\left(\left(\nu-r \rho+\frac{r x}{t^{r}}\right) t^{\nu-2 r \rho} e^{-x t^{-r}}\right)^{\prime} \cdot e^{-t^{\rho}} d t \\
=\left.\left(\nu-r \rho+\frac{r x}{t^{r}}\right) t^{\nu-2 r \rho} e^{-x t^{-r}} \cdot e^{-t^{\rho}}\right|_{0} ^{\infty} \\
\quad+\rho \int_{0}^{\infty}\left(\nu-r \rho+\frac{r x}{t^{r}}\right) t^{\nu-2 r \rho+\rho-1} e^{-x t^{-r}} e^{-t^{\rho}} d t \\
=\rho \int_{0}^{\infty}\left(\nu-r \rho+\frac{r x}{t^{r}}\right) t^{\nu-(2 r-1) \rho-1} e^{-x t^{-r}} e^{-t^{\rho}} d t .
\end{aligned}
$$

If we rewrite the expression in (65) relation as

$$
v-r \rho+\frac{r x}{t^{r}}=v-(2 r-1) \rho+\frac{r x}{t^{r}}+(r-1) \rho
$$

then we have

$$
\begin{gathered}
\left(\mathscr{T}_{\rho}^{\nu} Y_{\rho, r}^{\nu}\right)(x)=\rho \int_{0}^{\infty}\left(\nu-(2 r-1) \rho+\frac{r x}{t^{r}}\right) \\
\cdot t^{\nu-(2 r-1) \rho-1} e^{-x t^{-r}} e^{-t^{\rho}} d t+\rho^{2}(r-1) \\
\cdot \int_{0}^{\infty} t^{\nu-(2 r-1) \rho-1} e^{-x t^{-r}} e^{-t^{\rho}} d t .
\end{gathered}
$$

If we evaluate the integral on the right-hand side of relation (64) and apply the integration by parts, we arrive at (62) as follows:

$$
\begin{aligned}
\left(\mathscr{T}_{\rho}^{\nu} Y_{\rho, r}^{\nu}\right)(x)= & \rho \int_{0}^{\infty}\left(t^{\nu-(2 r-1) \rho} e^{-x t^{-r}}\right)^{\prime} e^{-t^{\rho}} d t \\
& +\rho^{2}(r-1) Y_{\rho, r}^{\nu-(2 r-1) \rho} \\
= & \left.\rho \cdot t^{\nu-(2 r-1) \rho} e^{-x t^{-r}} e^{-t^{\rho}}\right|_{0} ^{\infty} \\
& -\rho^{2} \int_{0}^{\infty} t^{\nu-2(r-1) \rho-1} e^{-x t^{-r}} e^{-t^{\rho}} d t \\
& +\rho^{2}(r-1) Y_{\rho, r}^{\nu-(2 r-1) \rho} \\
= & -\rho^{2} Y_{\rho, r}^{\nu-2(r-1) \rho}+\rho^{2}(r-1) Y_{\rho, r}^{\nu-(2 r-1) \rho},
\end{aligned}
$$

where

$$
\begin{aligned}
& \left(t^{\nu-(2 r-1) \rho} e^{-x t^{-r}}\right)^{\prime} \\
& \quad=\left(\nu-(2 r-1) \rho+\frac{r x}{t^{r}}\right) t^{\nu-(2 r-1) \rho-1} e^{-x t^{-r}}
\end{aligned}
$$

Remark 13. If $v \in \mathbb{C}$ and $\rho=1$, then the function $Y_{1,1}^{v}(x)=$ $Z_{1}^{v}(x)$ is a solution of the differential equation of fourth order

$$
x^{2} y^{(I V)}+(2 v-4) x y^{\prime \prime \prime}+(v-1)(v-2) y^{\prime \prime}+y=0
$$

(cf. $[13$, p. 21]).

\section{Conclusion}

Mejer's $G$ functions, which are generalization of hypergeometric functions, are Mellin-Barnes integrals. Generalized Krätzel functions, $Y_{\rho, r}^{v}(x)$ could be written in terms of $H$-functions, which are generalization of $G$-function, as a Mellin-Barnes integral. Furthermore, the integral transform with the kernel $Y_{\rho, r}^{v}(x)$ could be investigated.

\section{Competing Interests}

The authors declare that they have no conflict of interests regarding the publication of this paper.

\section{References}

[1] E. Krätzel, "Integral transformations of Bessel-type," in Generalized functions and operational calculus (Proc. Conf. Varna 1975), pp. 148-155, Bulgarian Academy of Science, Sofia, Bulgaria, 1979.

[2] A. A. Kilbas and D. Kumar, "On generalize krätzel function," Integral Transforms and Special Functions, vol. 20, no. 11, pp. 835-846, 2009.

[3] D. Kumar, "Some among generalized Krätzel function, Ptransform and their applications," in Proceedings of the National Workshop on Fractional Calculus and Statistical Distributions, pp. 47-60, CMS Pala Compus, November 2009.

[4] P. Turan, "On the zeros of the polynomials of Legendre," Casopis pro Pěstování Matematiky a Fysiky, vol. 75, no. 3, pp. 113-122, 1950.

[5] D. V. Widder, The Laplace Transform, Princeton University Press, Princeton, NJ, USA, 1941.

[6] D. S. Mitrinović, J. E. Pecaric, and A. M. Fink, Classical and New Inequalities in Analysis, Kluwer Academic Publishers, Dordrecht, The Netherlands, 1993.

[7] S. G. Samko, A. A. Kilbas, and O. I. M. Marichev, Fractional Integrals and Derivatives, Theory and Applications, Gordon and Breach S.P., New York, NY, USA, 1993.

[8] K. B. Oldham and J. Spainer, The Fractional Calculus, Mathematics in Science and Engineering, vol. 111, Academic Press, New York, NY, USA, 1974.

[9] L. Debnath, "Recent applications of fractional calculus to science and engineering," International Journal of Mathematics and Mathematical Sciences, no. 54, pp. 3413-3442, 2003. 
[10] F. Mainardi, Applications of Fractional Calculus in Mechanics, Transform Methods and Special Functions, Varna 96, Edited by P. Rusev, I. Dimovski, and V. Kiryakova, Bulgarian Academy of Sciences, Sofia, Bulgaria, 1998.

[11] R. K. Saxena and S. L. Kalla, "On a generalization of Kratzel function and associated inverse Gaussian distribution," Algebras, Groups, and Geometries, vol. 24, pp. 303-324, 2007.

[12] A. Baricz, D. Jankov, and T. K. Pogány, "Turán type inequalities for Krätzel functions," Journal of Mathematical Analysis and Applications, vol. 388, no. 2, pp. 716-724, 2012.

[13] B. Bonilla, M. Rivero, J. Rodriguez, J. Trujillo, and A. A. Kilbas, "Bessel-type functions and bessel-type integral transforms on spaces $F_{p, \mu}$ and," Integral Transforms and Special Functions, vol. 8, no. 1-2, pp. 13-30, 1999.

[14] A. Erdélyi, W. Magnus, and F. Oberhettinger, Tables of Integral Transforms, vol. 1, McGraw-Hill, New York, NY, USA, 1954.

[15] A. Erdélyi, W. Magnus, and F. Oberhettinger, Tables of Integral Transforms, vol. II, McGraw-Hill, New York, NY, USA, 1954. 


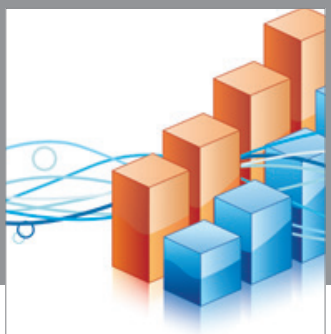

Advances in

Operations Research

vatem alat4

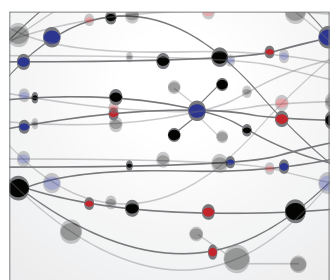

\section{The Scientific} World Journal
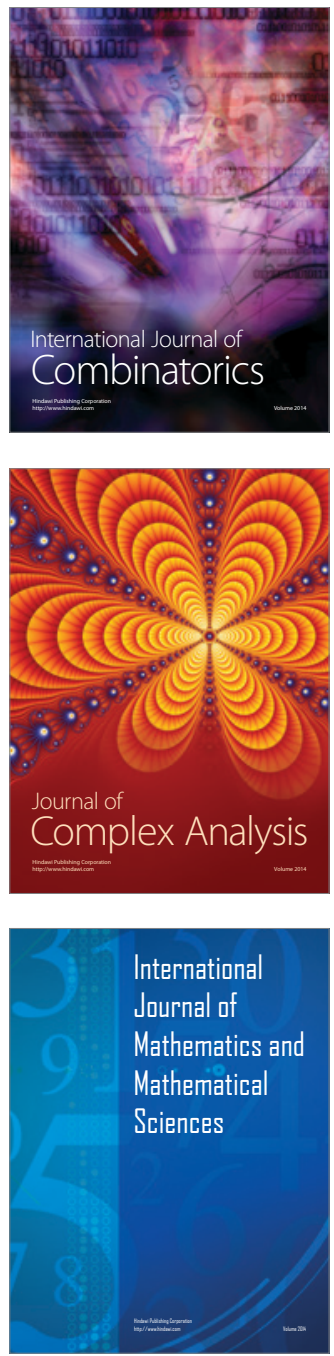
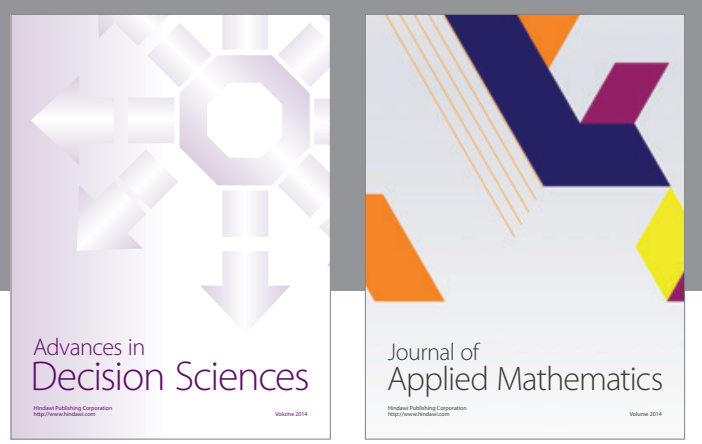

Algebra

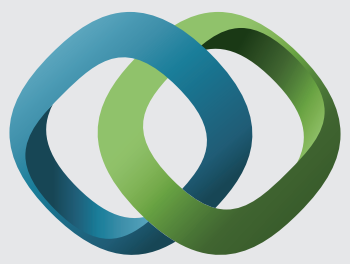

\section{Hindawi}

Submit your manuscripts at

https://www.hindawi.com
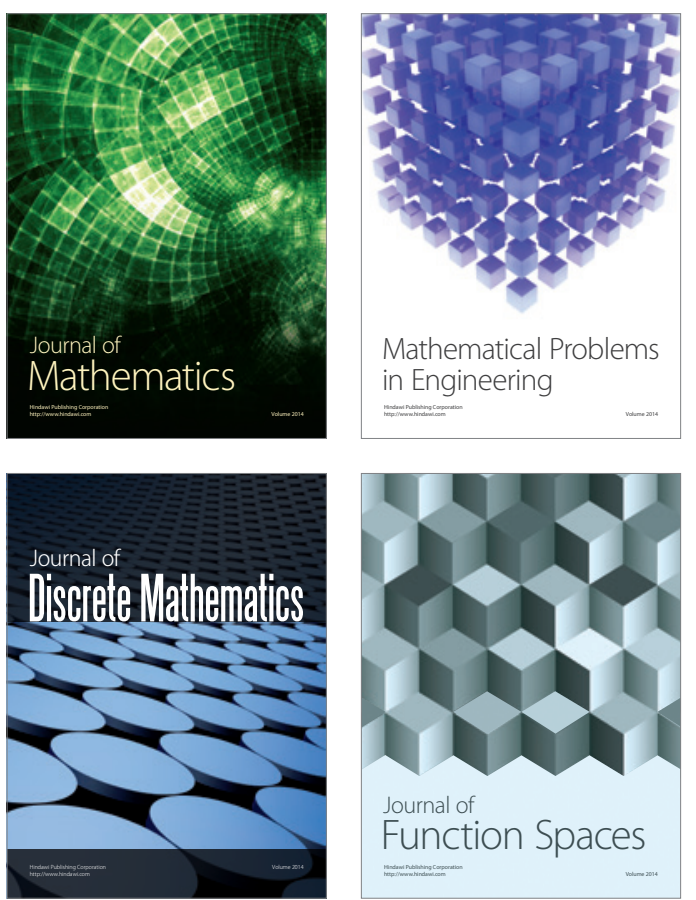

Mathematical Problems in Engineering
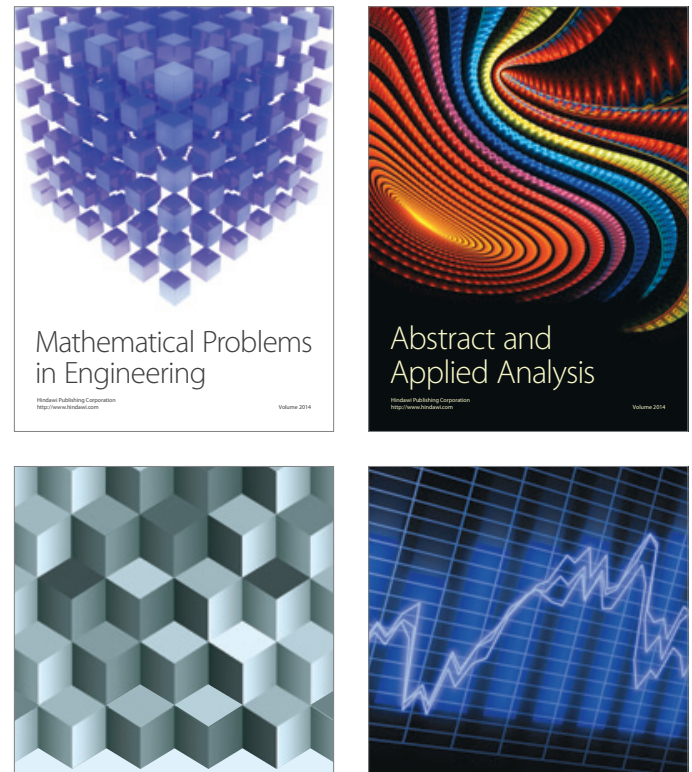

Journal of

Function Spaces

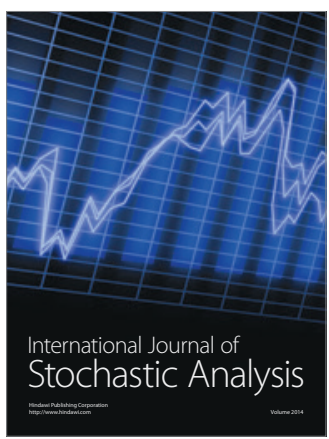

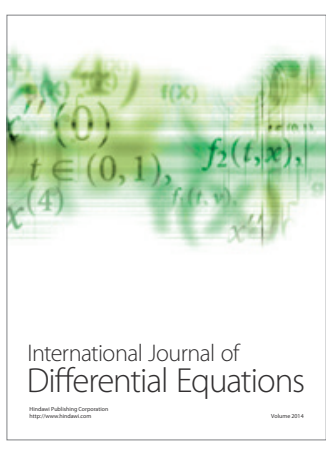
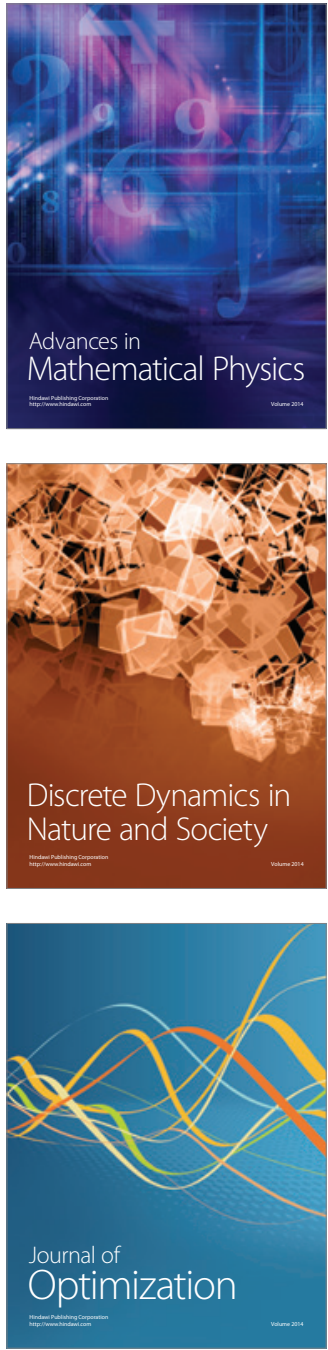